\title{
An Integrated Platform for Advanced Diagnostics
}

\author{
Giovanni De Micheli*, S. Sara Ghoreishizadeh*, Cristina Boero*, Francesco Valgimigli ${ }^{\dagger}$ and Sandro Carrara* \\ * EPFL, LSI - Lausanne - Switzerland \\ $\dagger$ A. Menarini Diagnostics - Florence - Italy \\ Email: giovanni.demicheli@epfl.ch
}

\begin{abstract}
The objective of this work is the systematic study of the use of electrochemical readout for advanced diagnosis and drug monitoring. Whereas to date various electrochemical principles have been studied and successfully tested, they typically operate on a single target molecule and are not integrated in a full data analysis chain. The present work aims to view various sensing approaches and explore the design space for integrated realization of multi-target sensors and sensor arrays.
\end{abstract}

Index Terms-biosensor, integrated circuit, metabolite, oxidase, cytochrome $\mathbf{P 4 5 0}$, potentiostat.

\section{INTRODUCTION}

Electrochemical detection of biological agents is important in several domains, including the medical practice, the discovery of biological mechanism and monitoring the environment. To date, several medical devices use electrochemical detection. A notable case is the family of FDA-approved devices for glucose monitoring. Most existing systems consists of the the combination of fluidic systems, sensors and electronic circuits. Our final objective is the creation of fully-integrated sensors. To achieve this goal, we focus our attention on integrating sensing and readout systems.

There are several examples of integrated biosensors. Several works deal with integrated chips for label-free DNA detection by using capacitance measurements [1], or by means of redox couples [2]. Integration is also the key-point to develop implantable biosensors for long-term monitoring of different compounds [3], [4], [5], [6]. In the medical practice, existing FDA-approved sensors are not fully integrated. A notable example is the GlucoMen $\cap$ Day, which can provide realtime measurements of subcutaneous glucose for up to 100 hours [7]. There are several architectures and technologies for integrated biosensors, and most devices are designed with ad hoc methods. Moreover the current need of multiple-target biosensing requires an extension of the existing techniques. Thus, the proliferation of electronic monitoring techniques would benefit from a systematic design space exploration, in the search of the most cost-effective solution (e.g., small, low energy consumption, low-cost) to a given problem. In this paper we propose the use of a platform, i.e., a restriction of the design space to the use of a small number of parametrized components, to cope with the design of integrated multipletarget biosensors.

978-3-9810801-7-9/DATE11/(C2011 EDAA

\section{A. Medical motivation}

Monitoring human metabolism is of crucial importance for personalized medicine. Many metabolic diseases may be monitored by means of different endogenous metabolites. On the other hand, metabolism of exogenous compounds can enhance the specificity of a therapy according to the individual patient, since the response rate of different patients to the same pharmacological treatment and dose typically varies in the range from $20 \%$ to $50 \%$. Bio-monitoring can be effectively used to tune the dosage of a therapy.

Among endogenous metabolites the most interesting are glucose, lactate, glutamate, and cholesterol. Glucose amount in the human blood is a marker for diabetes. Lactic acidosis is one of the effect of Von Gierke's disease and it occurs when mitochondria cannot satisfy cell demand of ATP, especially during physical efforts or diseases. Glutamate is a neurotransmitter, which can be found in neuronal and glial membranes. In brain injury or brain diseases, glutamate can accumulate outside cells, leading to neuronal damage and, eventually, cell death. Cholesterol is a lipid, and it is one important building block in the structure of cells. However, high levels in the blood are strongly associated to atherosclerosis.

Many exogenous metabolites are relevant molecules commonly used in drug therapy. Etoposide, ftorafur, cyclophospamide, ifosfamide, etc. are well known molecules typically used in chemotherapy treatments. The measure of their level in the blood during pharmacological therapy allows doctors to monitor how the patient is metabolizing the supplied drugs.

\section{B. Electrochemical principles}

Enzymes are very useful proteins, which may be used as probes to monitor metabolites in case of both endogenous and exogenous molecules. In particular, the protein class of oxidases may be used for sensing endogenous metabolites, while cytochromes P450 may be used for sensing drug compounds. The term oxidase is referred to a large family of enzymes that catalyzes an oxidation-reduction reaction, involving molecular oxygen $\left(\mathrm{O}_{2}\right)$ as the electron acceptor. In these reactions, the final products can be water $\left(\mathrm{H}_{2} \mathrm{O}\right)$, as well as hydrogen peroxide $\left(\mathrm{H}_{2} \mathrm{O}_{2}\right)$.

Oxidases are typically characterized by a prosthetic group integrated within the enzyme, which is the group directly involved in the redox reaction. For such enzymes like glucose oxidase (GOD), L-glutamate oxidase (GIOD), and cholesterol 
TABLE I

LIST OF OXIDASES USED TO DEVELOP BIOSENSORS .

\begin{tabular}{cccc}
\hline Oxidase species & $\begin{array}{c}\text { Target } \\
\text { molecule }\end{array}$ & Description & $\begin{array}{c}\text { Applied potential } \\
\text { (vs Ag/AgCl) }\end{array}$ \\
\hline \hline $\begin{array}{c}\text { GLUCOSE } \\
\text { OXIDASE }\end{array}$ & glucose & $\begin{array}{c}\text { Metabolic compound } \\
\text { as energy source }\end{array}$ & $+550 \mathrm{mV}[8]$ \\
\hline $\begin{array}{c}\text { LACTATE } \\
\text { OXIDASE }\end{array}$ & lactate & $\begin{array}{c}\text { Metabolic compound as } \\
\text { marker of cell suffering }\end{array}$ & $+650 \mathrm{mV}[9]$ \\
\hline $\begin{array}{c}\text { L-GLUTAMATE } \\
\text { OXIDASE }\end{array}$ & glutamate & Excitatory neurotransmitter & $+600 \mathrm{mV}[10]$ \\
\hline $\begin{array}{c}\text { CHOLESTEROL } \\
\text { OXIDASE }\end{array}$ & cholesterol & $\begin{array}{c}\text { Metabolic compound that } \\
\text { establishes proper membrane } \\
\text { permeability and fluidity }\end{array}$ & $+700 \mathrm{mV} \mathrm{[11]}$ \\
\hline
\end{tabular}

oxidase (COD), the prosthetic group is the flavin adenine dinucleotide (FAD) group. FAD can be reduced to $\mathrm{FADH}_{2}$ by accepting two hydrogen atoms. Then, the reduced form can be oxidized to restore the initial structure, combining hydrogen $\left(\mathrm{H}_{2}^{+}\right)$with $\mathrm{O}_{2}$ and loosing two electrons to produce hydrogen peroxide. The redox reaction for all these types of enzyme with FAD as prosthetic group is the following:

$$
\begin{gathered}
\mathrm{FAD}+\text { substrate } \rightarrow \mathrm{FADH}_{2}+\text { product } \\
\mathrm{FADH}_{2}+\mathrm{O}_{2} \rightarrow \mathrm{H}_{2} \mathrm{O}_{2}+\mathrm{FAD}
\end{gathered}
$$

Another subclass of enzymes (lactate oxidase, for example) employs flavin mononucleotide (FMN) group, which works in the same way as FAD. For both the prosthetic groups, the final product of the reaction is the hydrogen peroxide, which is an electrochemical active species. The following reaction

$$
2 \mathrm{H}_{2} \mathrm{O}_{2} \rightarrow 2 \mathrm{H}_{2} \mathrm{O}+\mathrm{O}_{2}^{+}+4 e^{-}
$$

is promoted by applying a potential to the electrochemical cell.

Cytochrome P450 (CYP) belongs to a family of heme proteins which metabolize over 200,000 chemicals, including drugs. Since the liver is the main biotransforming organ for drugs, CYPs and other metabolizing enzymes reside in the hepatocytes. As FAD and FMN were the active groups involved in the electron exchange for oxidase, the heme group has an analogous role for cytochromes. The most common redox reaction for this family of proteins is described as follows:

$$
\text { substrate }+\mathrm{O}_{2}+2 \mathrm{H}^{+}+2 e^{-} \rightarrow \text { product }+\mathrm{H}_{2} \mathrm{O}
$$

where the supplier of electrons is the heme group. Thus, it is possible to follow the catalytic cycle of cytochrome by monitoring the electron transfer between the electrode and the enzyme by using an amperometric biosensor. On this basis, it is possible to determine the concentration of the target molecule by measuring the current generated in the electrochemical cell. There are two main electrochemical techniques to measure target concentration, according to the biological agent used in the sensing mechanism.

The common product of oxidases is $\mathrm{H}_{2} \mathrm{O}_{2}$, as mentioned before. The oxidation of hydrogen peroxide is well known [22] and can be measured by setting the electrode potential at $+650 \mathrm{mV}$. For this reason it is possible to apply a con-

\begin{tabular}{|c|c|c|c|}
\hline $\begin{array}{c}\text { CYP } \\
\text { species }\end{array}$ & $\begin{array}{c}\text { Target } \\
\text { drug }\end{array}$ & Description & $\begin{array}{c}\text { Reduction potential } \\
\text { (vs } \mathrm{Ag} / \mathrm{AgCl})\end{array}$ \\
\hline CYP1A2 & clozapine & $\begin{array}{l}\text { Antipsychotic used in the } \\
\text { treatment of schizophrenia }\end{array}$ & $-265 \mathrm{mV}[12]$ \\
\hline CYP3A4 & $\begin{array}{l}\text { erythromicin } \\
\text { indinavir }\end{array}$ & $\begin{array}{l}\text { Broad-spectrum antibiotic } \\
\text { Used in the treatment } \\
\text { of HIV infection and AIDS }\end{array}$ & $\begin{array}{l}-625 \mathrm{mV}[13] \\
-750 \mathrm{mV}[14]\end{array}$ \\
\hline CYP11A1 & cholesterol & $\begin{array}{l}\text { Metabolite able to establish } \\
\text { proper cell membrane } \\
\text { permeability and fluidity }\end{array}$ & $-400 \mathrm{mV}[15]$ \\
\hline \multirow[t]{2}{*}{ CYP2B4 } & benzphetamine & $\begin{array}{l}\text { Used in the treatment } \\
\text { of obesity }\end{array}$ & $-250 \mathrm{mV}[16]$ \\
\hline & aminopyrine & $\begin{array}{l}\text { Analgesic, anti-inflammatory, } \\
\text { and antipyretic drug }\end{array}$ & $-400 \mathrm{mV}[17]$ \\
\hline \multirow[t]{2}{*}{ CYP2B6 } & bupropion & Antidepressant & $-450 \mathrm{mV}[18]$ \\
\hline & lidocaine & Anesthetic and antiarrhythmic & $-450 \mathrm{mV}[19]$ \\
\hline CYP2C9 & $\begin{array}{l}\text { torsemide } \\
\text { diclofecan }\end{array}$ & $\begin{array}{c}\text { Diuretic } \\
\text { Anti-inflammatory }\end{array}$ & $\begin{array}{l}-19 \mathrm{mV}[20] \\
-41 \mathrm{mV}[20]\end{array}$ \\
\hline CYP2E1 & p-nitrophenol & $\begin{array}{l}\text { Intermediate in the } \\
\text { synthesis of paracetamol }\end{array}$ & $-300 \mathrm{mV}[21]$ \\
\hline
\end{tabular}

TABLE II

LIST OF CYTOCHROMES USED TO DEVELOPED BIOSENSORS AND THEIR ELECTROCHEMICAL PARAMETERS.

stant potential, corresponding to the oxidation potential, and recording the current generated, which is directly proportional to the concentration of the target molecule metabolized by the oxidase. This technique is called chronoamperometry, because the current is monitored as a function of time. With screenprinted electrodes, the current decays over the time, and it reaches an approximately steady-state after a certain time.

Another technique used in electrochemistry is called cyclic voltammetry $(\mathrm{CV})$, and it is more suitable in the case of CYT P450, where it is possible to identify more target molecules with the same biological actuator. In fact, by using CV it is possible to identify multiple targets and their concentration at the same time. Cyclic voltammetry applies a linear-sweep potential forward and backward within a potential window to the electrochemical cell, and monitoring continuously the current. The resulting graph will rise very slowly at the beginning. As soon as the potential approaches the reduction potential, the electron transfer from the protein to the electrode increases its rate, which causes cell current to increase. At a certain point, the current will start to decrease again, due to the decreasing rate of diffusion of the target from the bulk of the solution. Typically, the current is plotted as function of the voltage and the plot is characterized by some peaks, whose height is proportional to the target concentration, while position gives information on the type of molecules that are oxidized, like an electrochemical signature. Table I summarizes some examples of oxidases used to develop biosensors, where chronoamperometry is used as the electrochemical technique used for the detection of hydrogen peroxide (applying a potential around $+650 \mathrm{mV}$ ). Table II reports some target molecules, detectable by CYP and their typical reduction potential.

\section{PhysiCAL DESIGN OF THE SENSOR}

Chronoamperometry and cyclic voltammetry require electrochemical cells consisting of three electrodes: i) a working 
electrode (WE), ii) a reference electrode (RE) and iii) a counter electrode (CE). A potentiostat circuit keeps the electric potential of the reference and working electrodes - as well as the interposed fluid - to a value that can be fixed or variable with respect to ground. The current that flows to ground through the working electrode is collected and measured.

When considering sensing multiple targets, it is possible to consider various bio-electrical interfaces. The simplest physical structure is a single sensor, made of 3 electrodes, with WE that is functionalized with enzymes to sense one or more targets. An extension of this sensor for $n$ targets, is to use $n+2$ electrodes, out of which there are $n$ working electrodes each functionalized for a given target. An underlying assumption for this sensor to be useful is that the targets can be present in the same volume surrounding the probe without interfering. A one-dimensional (or two-dimensional) sensor array consists of $k$ (or $k \times j$ ) such sensors, each with 3 or more electrodes. Finally, when the electrochemical reactions must be kept separated, each sensor in an array must have its own chamber.

\section{A. Sensor design for multiple targets}

In this section we want to consider jointly: i) the choice of the probe; ii) the choice of the sensor structure; iii) the choice of electronic readout circuitry. We start from the choice of the probe, as this is typically dictated by the target, and the target is often the objective of the biomedical investigation.

Let us consider first oxidase-based sensing. In this case, quantitative measures are obtained from the current generated by the oxidation of hydrogen peroxide. In a first approximation it is possible to combine more oxidases in the same chamber to detect different metabolites, even if all the oxidases generate current by oxidation of the hydrogen peroxide. Since the diffusion coefficient of $\mathrm{H}_{2} \mathrm{O}_{2}$ is really low, we can assume negligible cross-talk among the different sensors. Otherwise, we should consider separate chambers, where an array of electrochemical cells achieve the multitarget sensing.

Next, let us consider CYP-based sensing. As mentioned before, the simplest CYP measure consists in a cyclic voltammetry, i.e., by cycling the potential of the fluid interposed between the RE and WE, and by measuring the current flowing out of the WE. According to the cytochrome isoform chosen, one or more targets can be detected by current peaks corresponding to the related electrochemical potentials. Thus, a single probe can sense multiple targets, if the I/V plot can be recorded in correspondence with current maxima.

An alternative approach to multiple target detection can be achieved by sensors with more than one WE, each characterized by a different probe. In this case, cyclic voltammetry sweeps the potential of the fluid between RE and WE, and currents stemming out of the WEs will display peaks in correspondence with the related voltammetric potentials. A circuit sensing the corresponding currents possibly multiplexed - and their maxima is then needed. It is important to remark that the collected currents relate to the amount of target interacting with the probe, and thus multiple measures can be done in a single chamber.

The use of sensor arrays - with or without multiple chambers - is a straightforward extension. From an electronic standpoint, an issue is the ability to share hardware resources (such as voltage generators) and current sensors, possibly by multiplexing. As an example, De Venuto et al. showed how to multiplex the measurement of the currents coming from various probes, to sense concentrations of different molecules [23].

\section{B. Properties}

We list here the desirable properties of a biosensing acquisition chain.

- Limit of detection (LOD). It is the lowest quantity of a substance that can be distinguished from the absence of that substance (a blank value) within a stated confidence limit. The detection limit is estimated from the mean of the blank $\mathrm{V}_{b}$, the standard deviation of the blank $\sigma_{b}$, and some confidence factor. ACS committee on environmental improvement recommends the following definition to calculate LOD [24]:

$$
L O D=V_{b}+3 \sigma_{b}
$$

in order to have a definite risk of less than $7 \%$ for false positive.

- Sensitivity. It is the slope of the calibration curve, i.e. $\left(\mathrm{V}_{s s^{-}} \mathrm{V}_{b}\right)$ versus the analyte concentration, $\mathrm{c}$, or its logarithm [25], where $\mathrm{V}_{s s}$ is the steady-state response. Global sensitivity is due to the cascading effects of the i) electrochemical reaction, ii) electron collection, iii) current amplification and conversion. The average global sensitivity, $S_{a v g}$ can be defined as:

$$
S_{a v g}=\Delta V / \Delta C
$$

where $\Delta C$ is the measured range of analyte concentration and $\Delta V$ is the corresponding change in the output signal of the system.

- Linear concentration range and linearity. On the biosensor side, linear range is directly related to the biocatalytic or biocomplexing properties of the biochemical or biological receptor [25]. In the system level, the effects of the bioprobes membrane and electronics readout should be considered as well in limiting the linearity. The maximum non-linearity of a measurement platform can be defined as:

$$
N L_{\text {max }}=\max \left|V_{C}-V_{C_{0}}-S_{a v g} \cdot\left(C-C_{0}\right)\right|
$$

where $\mathrm{C}_{0}$ refers to a reference concentration, $\mathrm{V}_{C}$ and $\mathrm{V}_{C_{0}}$ are the output signals corresponding to $\mathrm{C}$ and $\mathrm{C}_{0}$ concentrations.

- Selectivity. It measures the ability to discriminate between different substances. Such behavior is principally a function of the recognition element, i.e. the enzymes [22]. 


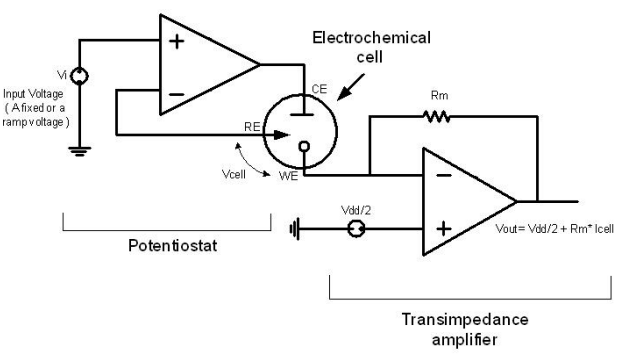

Fig. 1. A simple block diagram of a potentiostat and a transimpedance amplifier.

- Steady-state response time. It is the time necessary to reach $90 \%$ of the steady-state response. Transient response time corresponds to the time necessary for the first derivative of the output signal to reach its maximum value $(\mathrm{dV} / \mathrm{dt})_{\max }$ following the analyte addition. Both response times depend upon the analyte, co-substrate and product transport rates through different layers or membranes. They also depend upon the activity of the molecular recognition system and the mixing conditions of the sample into the batch measurement cell [25]. Since the biochemical sensing is a very slow process, the readout circuitry would not limit the response times of the output signal.

- Sample throughput. It is a measure of the number of individual samples per unit of time. This parameter takes into account both the transient response time and the recovery time, i.e. the time needed for the signal to return to its baseline [25].

In the sequel, we concentrate on the following parameters: 1) limit of detection, 2) sensitivity, 3) linearity and 4) time response, because they affect the design of the electronic building blocks.

\section{Electrical building blocks for biosensing}

We restrict our attention to biosensing achieved by oxidases and CYP by means of observing a current while controlling potentials.

Major building blocks are:

- A current readout circuit that can sense currents in the range of $+/-10 \mathrm{uA}$ with $10 \mathrm{nA}$ resolution for oxidases, and the range of $+/-100 \mathrm{uA}$ with $100 \mathrm{nA}$ resolution for CYP, and translate it into a voltage that can be digitized through an ADC.

- A potentiostat circuit that has the purpose of keeping the reference and working electrode, as well as the surrounded fluids, at a given potential with respect to ground. One of the possible potentiostat configurations is shown in Fig. 1.

- A voltage generator that generates a fixed or variable voltage to feed the potentiostat circuit. For single-target chronoamperometry, the voltage is fixed and chosen on the basis of the electrochemical reaction. For cyclic voltammetry, this circuit sweeps repeatedly within the voltage range of interest.

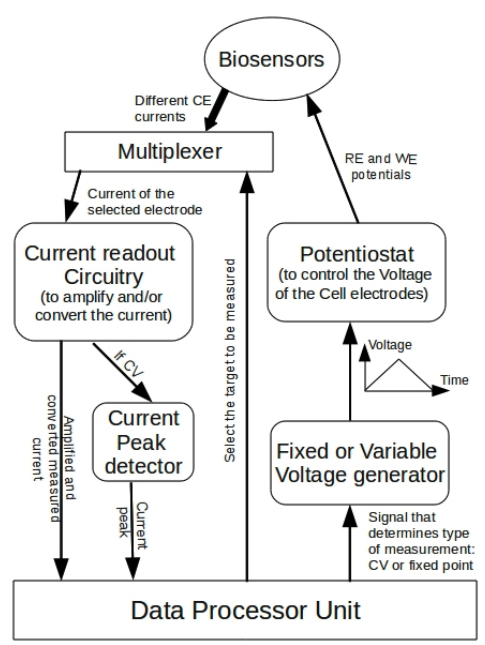

Fig. 2. Building block diagram for a biosensing platform.

- Multiplexing circuits to support the readout of multiple current sources and the drive of multiple control points for the potential.

A building block diagram for a biosensing platform is shown in Fig. 2.

Different approaches to readout can be used. We describe here the fundamental principles and their limitations. The most straightforward approach is to convert the biosensor current into voltage using a transimpedance amplifier as shown in Fig. 1. As the current levels are low, specific techniques can be used such as using current mirrors, multi-stage amplification and/or switched-capacitor circuits. Alternative approaches convert currents to the frequency domain [26], [27] .

The amplification stage has to satisfy some characteristics, such as linearity, step response and low-noise. Whereas all these are typical of data acquisition schemes, particular attention has to be given to noise issues. The sensor noise is hard to quantify analytically, but it can be measured experimentally. The amplifier input noise has to be made negligible as compared to the sensor node. Particular care has to be taken for the Flicker (or $1 / f$ ) noise component, which can be reduced by techniques such as chopping and Correlated Double Sampling (CDS). Chopping involves moving the signal of interest to a higher frequency before amplification. In CDS, the output of the sensor is measured twice: once in a known condition and once in an unknown condition. The value measured from the known condition is then subtracted from the unknown condition to generate a value with a known relation to the molecule concentration being measured. The latter can be realized using an extra WE without any enzyme on it. Note that the extra WE is not helpful in presence of molecules such as Dopamine and Etoposide, as they can oxidize by applying a voltage to the WE even without any enzyme.

In cyclic voltammetry, the rate of voltage variation affects the accuracy of measurement. The electrochemical cell reacts only to slow potential variations of about $20 \mathrm{mV} / \mathrm{sec}$. If the voltage changes too rapidly, the biosensor current peak does 


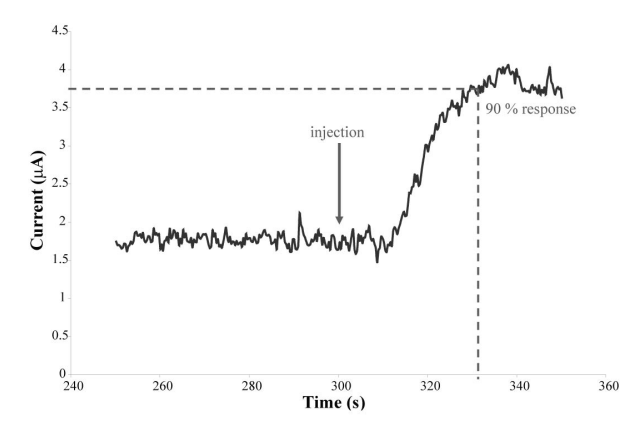

Fig. 3. Time-response example for a glucose biosensor.

not occur at the specific potential of the target molecule anymore, making it hard to distinguish among different targets. An example of time response for a glucose biosensor is depicted in Fig. 3. We can notice that the signal takes around 30 seconds to reach the steady-state after an injection of the target molecule. As data acquisition deals specifically with slow signals in our case, noise and drift control (e.g. stability) at low frequencies is very important.

\section{A PLATFORM EXAMPLE}

Different strategies have been proposed to develop integrated biosensors. The most common and widely used foresees to use cheap disposable electrodes, which can be inserted in a separate device with the integrated electronics [28], [29]. This approach is unsuitable for long-term implants in the body. Another technique is to fabricate 3-D integration chips with a top replaceable bio-layer, as showed by Temiz et al. [30]. Despite these systems are not yet implantable, it is possible to jointly fabricate electrodes and electronic circuits to reduce costs. The third approach involves disposable electrodes and electronics, which can be thrown away once a series of measurements is performed. An example is the Quicklabß system by Siemens.

Figure 4 shows the biointerface of our platform. Scaling down the electrodes can bring some advantages: the background current is smaller, due to different double-layer capacitance phenomena[22]; time response of the biosensor is decreased in the case of microelectrodes, enabling much shorter measurements [22]. In the proposed configuration, the different working electrodes share the same counter and reference electrodes, so it is necessary to multiplex the signal of the working electrodes, in order to activate them sequentially.

The bio-interface layer consists of five working electrodes, one counter and one reference electrode. Working and counter electrodes are made of a thin film of gold, while reference electrode is made of silver, both metals deposited on silicon by metal evaporation. A layer of $\mathrm{SiO}_{2}$ is used as passivation on the top of the electrical connections. The electrode area is 0.23 $\mathrm{mm}^{2}$, but can be further decreased. Electrical connection with the rest of the instrumentation is guaranteed by the pads, which match with an out-of-shelf interface. The whole device can be further miniaturized. Working electrodes can be functionalized

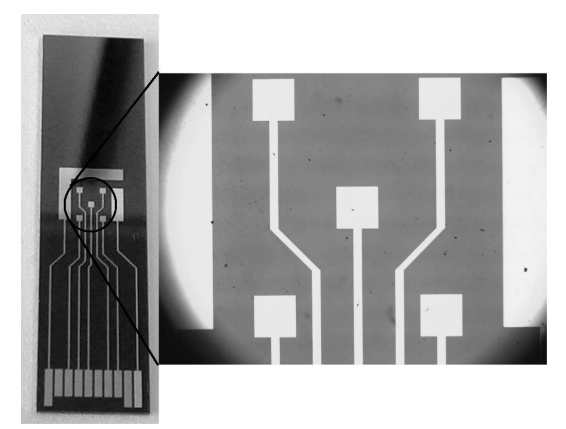

Fig. 4. An example of platform with multi-working electrodes.

by nanostructures, to increase sensitivity [15]; by polymers, to provide long-term stability [3]; and by the enzyme probe to enhance selectivity.

To achieve multi-panel metabolite detection, we can functionalize the 5 working electrodes to detect glucose, lactate, glutamate, benzphetamine, or aminopyrine, and cholesterol. Oxidases can be the probe for the first three compounds, while cytochromes are able to detect the other compounds (as already discussed in Table II). Note that with the same agent (CYP2B4) it is possible to detect different compounds (benzphetamine and aminopyrine) at the same electrode, due to the fact that the two molecules oxidize at different potential. The hight of the two corresponding peaks gives information about their concentration. Taking into account the discussion in Section II-B, Table III resumes the main properties of each functionalized electrode. Regarding glucose, lactate and glutamate detection, the values reported in the table are obtained in the case of single working electrodes nanostructured with carbon nanotubes. Benzphetamine and aminopyrine are measured on rhodium graphite electrodes [16], while cholesterol onto carbon nanotubes-based electrode [15]. All the mentioned functionalization and nanostructuration protocols can be easily integrated onto the developed platform and achieved by proteomic spotting.

With the present configuration, it is necessary to employ a fixed or variable voltage generator, according to the functionalization of each electrode. We need also a multiplexer, which switches sequentially among the different working electrodes. Finally, the current readout depends also on the type of functionalization, since oxidases do not need a current peak detector. Note from Table III that the introduction of a nanostructuration on the electrodes brings much larger signals, demanding less constrains for the readout circuit. On the other hand, benzphetamine and aminopyrine have a much lower sensitivity with respect to the other values, which can be further enhance by employing nanostructured electrodes.

\section{CONCLUSION}

The aim of the present work is to consider the different aspects for the development of a fully integrated platform for the detection of endogenous and exogenous metabolites for advanced diagnostics. Sensing multiple targets is of crucial importance for personalized therapy. We have resumed the 
TABLE III

PERFORMANCE OF DIFFERENT METABOLITE BIOSENSORS.

\begin{tabular}{ccccc}
\hline Target & Probe & $\begin{array}{c}\text { Sensitivity } \\
\left(\mu \mathrm{A} /\left(\mathrm{mM} \mathrm{cm}^{2}\right)\right)\end{array}$ & $\begin{array}{c}\text { Limit of } \\
\text { detection }(\mu \mathrm{M})\end{array}$ & $\begin{array}{c}\text { Linear } \\
\text { range }(\mathrm{mM})\end{array}$ \\
\hline \hline GLUCOSE & $\begin{array}{c}\text { glucose } \\
\text { oxidase }\end{array}$ & 27.7 & 575 & $0.5-4$ \\
& $\begin{array}{c}\text { lactate } \\
\text { oxidase }\end{array}$ & 40.1 & 366 & $0.5-2.5$ \\
\hline LACTATE & glutamate & 25.5 & 1574 & $0.5-2$ \\
\hline GLUTAMATE & oxidase & & 200 & $0.2-1.2[16]$ \\
\hline BENZPHETAMINE & CYP2B4 & 0.28 & 400 & $0.8-8[16]$ \\
AMINOPYRINE & 2.8 & - & $0.01-0.08[15]$ \\
\hline CHOLESTEROL & CYP11A1 & 112 & & \\
\hline
\end{tabular}

electrochemical principles for oxidases and cytochromes, two enzyme families which can detect the majority of human metabolites. We have reviewed different sensor configurations according to the different strategies of detection. We have analyzed the desirable properties of a biosensing acquisition chain. We have presented an electrical building block for multiple metabolite detection, discussing the implications between a given design and biosensor features. Finally, we have described the biointerface of a platform for the detection of endogenous and exogenous compounds.

\section{ACKNOWLEDGMENT}

The authors would like to thank C. Baj-Rossi for discussion on CYP mechanisms. The research has been funded in part by the project NanoSys, within the program ERC-2009-ADG246810, the SiNo-Swiss cooperation project IZLCZ2123967, the i-IronIC project that is financed with a grant from the Swiss Nano-Tera.ch initiative and evaluated by the Swiss National Science Foundation.

\section{REFERENCES}

[1] C. Stagni et al., "CMOS DNA sensor array with integrated A/D conversion based on label-free capacitance measurement," IEEE Journal of Solid State Circuits, vol. 41, pp. 2956-2965, 2006.

[2] M. Schienle et al., "A fully electronic DNA sensor with 128 positions and in-pixel A/D conversion," IEEE Journal of Solid-State Circuits, vol. 39, no. 12, pp. $2438-2445,2004$.

[3] D. A. Gough et al., "Function of an implanted tissue glucose sensor for more than 1 year in animals," Science Translational Medicine, vol. 2, p. 42ra53, 2010.

[4] Y.-C. Tsai et al., "Fabrication process of integrated multi-analyte biochip system for implantable application," in IEEE 22nd International Conference on Micro Electro Mechanical Systems, 2009, pp. $204-207$.

[5] A. Rahman et al., "Fabrication and packaging of a dual sensing electrochemical biotransducer for glucose and lactate useful in intramuscular physiologic status monitoring," IEEE Sensors Journal, vol. 9, no. 12, pp. $1856-1863,2009$.

[6] A. Guiseppi-Elie et al., "Design of a subcutaneous implantable biochip for monitoring of glucose and lactate," IEEE Sensors Journal, vol. 5, no. 3 , pp. $345-355,2005$.

[7] F. Valgimigli et al., "Evaluating the clinical accuracy of glucomenday: A novel microdialysis-based continuous glucose monitor," Journal of Diabetes Science Technology, vol. 4, pp. 1182-1192, 2010.

[8] M.-C. Tsai and Y.-C. Tsai, "Adsorption of glucose oxidase at platinummultiwalled carbon nanotube-alumina-coated silica nanocomposite for amperometric glucose biosensor," Sensors and Actuators B: Chemical, vol. 141, no. 2, pp. 592 - 598, 2009.
[9] M. R. Romero et al., "Design and optimization of a lactate amperometric biosensor based on lactate oxidase cross-linked with polymeric matrixes," Sensors and Actuators B: Chemical, vol. 131, no. 2, pp. 590 $-595,2008$.

[10] Y. Hu et al., "Direct measurement of glutamate release in the brain using a dual enzyme-based electrochemical sensor," Brain Research, vol. 659, no. 1-2, pp. $117-125,1994$.

[11] A. Salimi et al., "Fabrication of a sensitive cholesterol biosensor based on cobalt-oxide nanostructures electrodeposited onto glassy carbon electrode," Electroanalysis, vol. 21, no. 24, pp. 2693-2700, 2009.

[12] M. Antonini et al., "Preliminary electrochemical characterisation of cytochrome P4501A2 - clozapine interaction," IEE Proceedings on Nanobiotechnology, vol. 150, no. 1, pp. 31-34, 2003.

[13] N. R. Hendricks et al., "Microsomal cytochrome P450-3A4 (CYP3A4) nanobiosensor for the determination of 2,4-dichlorophenol-an endocrine disruptor compound," Electrochimica Acta, vol. 54, no. 7, pp. 19251931, 2009.

[14] A. Ignaszak et al., "Novel therapeutic biosensor for indinavir-a protease inhibitor antiretroviral drug," Journal of Pharmaceutical and Biomedical Analysis, vol. 49, no. 2, pp. 498-501, 2009.

[15] S. Carrara et al., "Screen-printed electrodes based on carbon nanotubes and cytochrome p450scc for higlhly sensitive cholesterol biosensors," Biosensors and Bioelectronics, vol. 24, pp. 148-150, 2008.

[16] V. V. Shumyantseva et al., "A new format of electrodes for the electrochemical reduction of cytochromes p450," Journal of Inorganic Biochemistry, vol. 100, no. 8, pp. 1353-1357, 2006.

[17] _ _ "Direct electron transfer of cytochrome P450 2B4 at electrodes modified with nonionic detergent and colloidal clay nanoparticles," Analytical Chemistry, vol. 76, no. 20, pp. 6046-6052, 2004.

[18] S. Liu et al., "Electrochemistry of cytochrome P450 enzyme on nanoparticle-containing membrane-coated electrode and its applications for drug sensing," Analytical Biochemistry, vol. 375, no. 2, pp. 209-216, 2008.

[19] L. Peng et al., "Electrochemistry of cytochrome P450 2B6 on electrodes modified with zirconium dioxide nanoparticles and platin components," Electroanalysis, vol. 20, no. 7, pp. 803-807, 2008.

[20] D. Johnson et al., "Electrochemical characterisation of the human cytochrome P450 CYP2C9," Biochemical Pharmacology, vol. 69, no. 10, pp. 1533-1541, 2005.

[21] A. Fantuzzi et al., "Direct electrochemistry of immobilized human cytochrome P450 2E1," Journal of the American Chemical Society, vol. 126, no. 16, pp. 5040-5041, 2004.

[22] B. R. Eggins, Chemical sensors and biosensors, LTD, Ed. John Wiley \& sons, pp. 125-169.

[23] D. De Venuto et al., "A novel multi-working electrode potentiostat for electrochemical detection of metabolites," in Proceedings of the IEEE Sensors Conference, 2010.

[24] D. MacDougall, "Guidelines for data acquisition and data quality evaluation in environmental chemistry," ACS COMMITTEE ON ENVIRONMENTAL IMPROVEMENT, Tech. Rep., 1980.

[25] D. R. Thevenot et al., "Electrochemical biosensors: recommended definitions and classification," IUPAC, Tech. Rep., 1999.

[26] M. Ahmadi and G. Jullien, "A very low power CMOS potentiostat for bioimplantable applications," in Fifth International Workshop on Systemon-Chip for Real-Time Applications, 2005, pp. 184 - 189.

[27] H. Narula and J. Harris, "A time-based VLSI potentiostat for ion current measurements," IEEE Sensors Journal, vol. 6, no. 2, pp. 239 - 247, 2006.

[28] Z. Chen et al., "Disposable glucose test strip for whole blood with integrated sensing/diffusion-limiting layer," Electrochimica Acta, vol. 55, pp. 544-550, 2009.

[29] W.-C. Shih et al., "Development of disposable lipid biosensor for the determination of total cholesterol," Biosensors and Bioelectronics, vol. 24, pp. 1679-1684, 2009.

[30] Y. Temiz et al., "3D architecture and replaceable layers for label-free DNA biochips," in 3rd International Workshop on Advances in Sensors and Interfaces, 2009, pp. $35-40$. 